\title{
Preliminary Study on the Assessment of Patients with Impaired Visuospatial Ability in Three-Dimensional Space Using a New Head Mounted Display System with Virtual Reality Technology
}

\author{
Toshiaki Tanaka, Norio Kato, Mizuki Yagi , Nobuya Hashimoto, Akira Kudo, Syunichi Sugihara
}

\begin{abstract}
Various disturbances of visual space recognition include unilateral spatial neglect (USN) has been strongly associated with an increased risk for injury and with poor functional outcome. The purpose of this study was to understand more accurately the pathology of USN using a new HMD system with virtual reality technology. In addition, brain activity measurements and analysis by using HMD in this study were done using near infrared spectroscopy (NIRS). Two experiments were performed as follows; (a) Experimental A (Object cancellation evaluation by using HMD): Unlike the line cancellation test in the BIT that one test used in the clinical evaluation of USN, in this case we tried to assess USN by conducting a test in which the task was to cancel three-dimensional objects using HMD. In this study, the respective USN symptoms in the egocentric and object-centered coordinate systems were evaluated. The objects were placed at depths of $35 \mathrm{~cm}, 45 \mathrm{~cm}$, and $55 \mathrm{~cm}$. The subject was a stroke patient (patient I ) with USN who was 83 years old. (b) Experimental B (Brain activity measurement and analysis during task execution): The subject was a stroke patient (patient II ) with USN who was 67 years old. The same task as in Experiment $I$ is performed, and the brain activity during the task movement is measured using NIRS. The subject took a resting chair, rested for 5 minutes, and then started the task. The NIRS measurement sites were from the inferior frontal gyrus to the middle frontal gyrus of both left and right sides. According to the results of in the experiment $A$, the cancellation rate showed the same trend that the left area was lower than central area and right area. The cancellation rate was the lowest at the greatest of the three depth distances, $55 \mathrm{~cm}$. This can only be seen with three-dimensional VR space as in this experiment, as scoring cannot be done on the BIT line cancellation test since tests related to depth cannot be performed. In the experiment $B$, greater values of oxygenated hemoglobin $(0 x y-\mathrm{Hb})$ related to brain activity was seen during the task than at rest in the patient with USN. Furthermore, the patient's brain activity on the
\end{abstract}

Toshiaki Tanaka, Faculty of Health Sciences, Hokkaido University of Science, Sapporo, Japan. / The Research Center for Advanced Science and Technology/ Institute of Gerontology, The University of Tokyo, Tokyo, Japan

Norio Kato, Faculty of Health Sciences, Hokkaido University of Science, Sapporo, Japan

Mizuki Yagi, Faculty of Health Sciences, Hokkaido University of Science, Sapporo, Japan.

Nobuya Hashimoto, Faculty of Health Sciences, Hokkaido University of Science, Sapporo, Japan.

Akira Kudo, Sapporo Shuyukai Hospital, Sapporo, Hokkaido, Japan

Syunichi Sugihara, Sapporo Shuyukai Hospital, Sapporo, Hokkaido, Japan paralyzed side was lower than the healthy side. In conclusions, the results suggest the possibility of analyzing versatile pathological conditions of USN with newly HMD system. Moreover, the results of NIRS suggested that it may also be to clarify brain activity in relation to the pathological condition of USN.

Index Terms - unilateral spatial neglect, virtual reality technology, head mounted display system.

\section{INTRODUCTION}

According to the Ministry of Health, Labour and Welfare, there were about 1.115 million cerebrovascular disease patients in Japan in 2017 [1]. One of the complications of cerebrovascular disease is unilateral spatial neglect (USN). USN is defined as "the inability to respond, react, and see the direction of stimuli presented on the side contralateral to the affected cerebral hemisphere"5[2]. It is a neurological syndrome with predilection to right hemisphere damage. The incidence of left USN, in which neglect symptoms are exhibited in the left space, is high, occurring in about $40 \%$ of patients in the acute stage [3]. Impairments in activities of daily living also originate from USN, in which things such as not braking the left side of wheelchairs, not putting the left foot on the footrest, and bumping into the wall on the left sometimes occur. This leads to an increased care burden, and USN is a major factor that impedes return to a role in society. Post-stroke USN is associated with older age and it is thought that the number of USN patients will also increase as society ages [4].

From the complexity of symptoms, the focal lesion in USN is also thought to vary widely. In 1941, Brain reported that the posterior parietal lobe is the location of lesions responsible for USN [5]. Since then, it has been said that impairments in the inferior parietal lobule, temporal junction, and frontal lobe, as well as regions from the superior temporal gyrus to the middle temporal gyrus and thalamus or reticular formation and their junctions, are also related to the occurrence of USN [6]. In order to determine the responsible lesion of USN is important to measure a brain activity, because it is deeply relation to accurate diagnosis and effective treatment for USN. Near infrared spectroscopy (NIRS) captures the changes in hemoglobin concentration accompanying brain blood flow changes that are dependent 


\section{Preliminary Study on the Assessment of Patients with Impaired Visuospatial Ability in Three-Dimensional Space Using a New Head Mounted Display System with Virtual Reality Technology}

on neural activity in the brain. Oxygenated hemoglobin (oxy-Hb) is considered the best indicator of blood flow changes [7]. Therefore, oxy-Hb would be taken to be the indicator of brain activity during these evaluation and training for USN.

USN is divided into several categories from the differences in its pathology. A typical one is categorization with the coordinate theory. The coordinate theory uses two spatial coordinate systems by which humans recognize space. One is a viewer-centered (egocentric) coordinate system in which the viewer's own body (neck, trunk, eyes) is the center of the coordinate system, and the other is an object-centered coordinate system centered on the objects that are being gazed upon [8]. One test used in the clinical evaluation of USN, the Behavioral Inattention Test (BIT), is a comprehensive evaluation method used internationally [9]. It consists of a conventional test and a behavioral test. The conventional test is a typical paper test that consists of six items, including cancellation, line bisection, copying, and drawing tasks. The behavioral test is evaluated with a method that mimics situations in activities of daily living. A maximum total score of 146 points, and nine behavioral subtests evaluating aspects of daily life, with a maximum total of 81 points. For a patient to be considered as exhibiting USN, the value of the conventional BIT should be less than 129, and lower rating values indicate worse USN. The Catherine Bergego Scale (CBS) is used to assess actual behavior in activities of daily living. A total of 10 items are assessed on a four-point scale of $0-3$. Of the possible total of 30 points, scores of 1-10 are taken to be mild neglect, 11-20 to be moderate neglect, and 21-30 to be severe neglect. The reliability and validity of the CBS have been verified in various studies, and it has sensitivity superior to tests done sitting at a desk [10].

Head mounted displays (HMDs) are widely used as devices to display the visual information used in virtual reality (VR) technology. They have begun to be widely adopted in the medical field, sports, and games. Trials of the use of HMDs in assessing USN have also been conducted. Tatiana et al., taking advantage of the feature of VR of being able to easily create various situations, developed a USN assessment based on virtual space in shopping situations, one of the activities of daily living [11]. Tanaka et al. developed a USN assessment based on coordinate theory using HMD and reported that it was also advantageous in improving the neglect detection rate [12]. However, while the object-centered coordinate condition could be created, the USN assessment itself was two-dimensional.

In this study, we developed and tested an assessment tool that elucidated impairment characteristics in three

dimensional space associated with depth. Using a program that assesses object search in virtual three dimensional space with an HMD and VR technology, the utility of this assessment tool for patients exhibiting USN was verified. In addition, brain activity measurements and analysis in the egocentric and object-centered coordinate conditions using HMD in this study were done using near infrared spectroscopy (NIRS).

\section{MATHODS}

2.1 Experiment A: Characteristics of the developed system

Firstly, we developed a tool which solid objects with pseudo-depth are placed using binocular parallax by use of VR technology. Quantitative three-dimensional assessment is done by having the objects disappear when touched by the controller. Moreover, by presenting images that reproduced the egocentric and object-centered coordinate systems in VR space, assessment was possible with two coordinate conditions in this study. This assessment tool also makes it possible to measure eye and head motion, and to analyze the movement of the eyes and head when an object is cancelled.

\subsubsection{Three-dimensional spatial assessment using VR}

The device used was an HMD (HTC; VIVE Pro Eye) (Figure 1). The advantages of using an HMD are that it can be implemented in a small space indoors for clinical use, and that an environment can be created in which the peripheral visual field is restricted and the subject can concentrate on the display within the HMD only. The assessment application was created using the Unity engine (2019.1.1f1). The content involves can-celling from the space multiple objects that have been placed there using a hand-held controller. In this assessment application, by cancelling three-dimensional objects that are placed arbitrarily on a three-dimensional grid, it is possible to quantitatively grasp the cancelled position in space

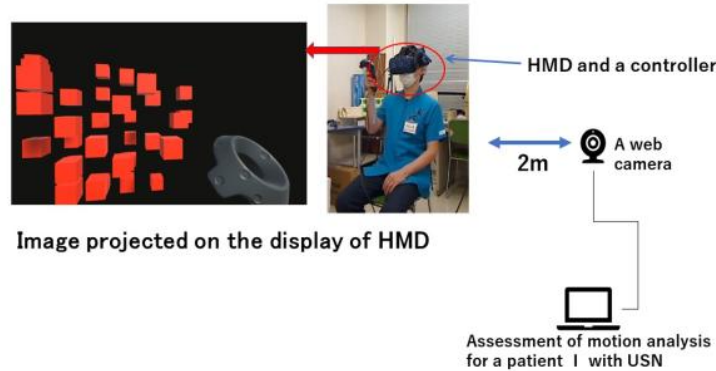

Fi. 1 Experiment $A$ :evaluation of USN by use of HMD.

\subsubsection{Assessment contents based on coordinate system theory}

It is possible to reproduce conditions in which the position of the object does not change with respect to the head even when the head moves (egocentric coordinates), and situations in which the same scene is viewed and the focus is always on the objects even when the head moves (object-centered coordinates). It is also possible to measure the time changes in head movement and eye movement. For head movement, the rotation movement of the head was captured using an internal gyroscope in the HMD, and for eye movement the line of sight was measured using an internal infrared sensor in the HMD.

2.1.3 Procedure of object cancellation test using HMD

Unlike the line cancellation test in the BIT, in this case we tried to assess USN by conducting a test in which the task was to cancel three-dimensional objects using HMD. 


\subsubsection{Subject}

The subject was a stroke patient (patient I ) with USN. The patient I was 83 years old who had undergone USN assessment with BIT and CBS in a co-operating medical institution. This study was approved by the ethical review board of The University of Tokyo (Review no. 11-23). The subject was fully informed about the study in advance, and his written consent was obtained before performing the measurements.

\subsubsection{Experiment environment with VR}

The experimental environment is shown in Figure 1. The experiment was per-formed in a private room isolated from the surroundings. The subjects were in a chair sitting position. During the experiment, the subjects wore an HMD on their head and were asked to hold the controller in their dominant hand (if they had paralysis, on the non-paralyzed side). To record video during the experiment, a web camera was placed in front of the subjects (about $2 \mathrm{~m}$ away). An infrared sensor (base station) was placed diagonally to measure the spatial coordinates of the HMD, controller, and other things.

\subsubsection{Object placement during object cancellation test}

In this study, the respective USN symptoms in the egocentric and object-centered coordinate systems were evaluated. Depth in this study was the horizontal distance, with the midpoint of the line connecting the left and right eyes taken as the origin. As shown in Figure 2, the objects were placed at depths of $35 \mathrm{~cm}, 45 \mathrm{~cm}$, and $55 \mathrm{~cm}$.

The objects presented in virtual three-dimensional space were placed evenly on six latitudinal and six longitudinal rows, three rows deep, within the range of the visual field, for a total of 108 objects (Figure 2). For the distance between the individual objects, the coordinates were input so that the objects would be placed evenly. The 36 objects in each depth row were place randomly. The objects were red cubes with the same shape.

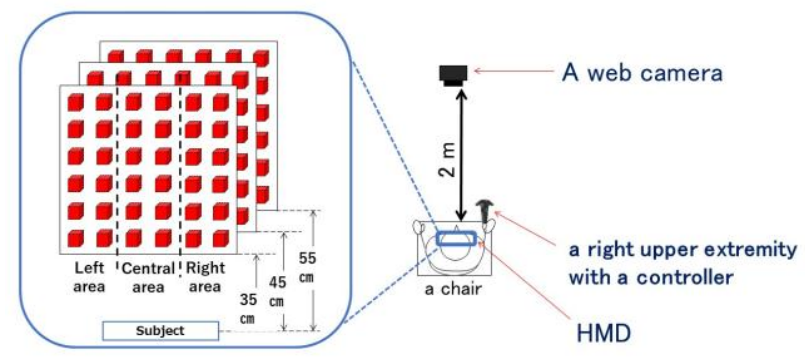

Fig. 2 The measuremental environment of experimental A: Object placement during object cancellation test

\subsection{Experimental B}

\subsubsection{Subject}

The subject was a patient (patient II ) with USN. The patient $I$ included patients in 67 years old who had undergone USN assessment with BIT and CBS in a cooperating medical institution. This study was also approved by the ethical review board of The University of Tokyo (Review no. 11-23).

\subsubsection{Experimental procedure}

The same task (object cancellation test at the object centered coordinate and the body centered coordinate.) as in Experiment $\mathrm{I}$ is performed, and the brain activity during the task movement is measured using NIRS (OCTAMON manufactured by Artinis Medical Systems). The task was repeated 4 times, and the time for each task was 5 minutes, and a break was taken between tasks for 1 minute. The subject took a resting chair, rested for 5 minutes, and then started the task. NIRS measurement sites were from the inferior frontal gyrus to the middle frontal gyrus of both left and right sides (Figure 3). The sampling rate at the time of data collection by NIRS was $50 \mathrm{~Hz}$.

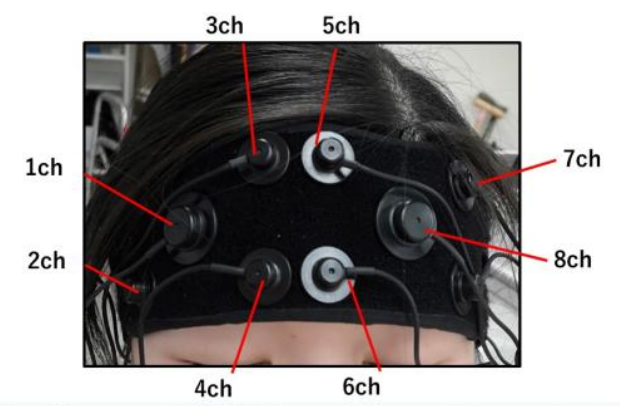

Fig. 3 Placement of eight measurement channels of near infrared spectroscopy (NIRS) at the frontal lobe.

\subsection{Analytical methods}

2.3.1 Experimental A : Object cancellation analysis

The objects placed were divided into three regions and analyzed. As shown in Figure 2, objects placed at the three depth distances $(35,45,55 \mathrm{~cm})$ were divided into a right area, middle area, and left area for each distance. From the number of objects placed in each area, the proportion derived by dividing by the number of cancelled objects was defined as the cancellation rate. Also, in analyzing head and eye movement, the total of the head movement and eye movement was defined as the gaze movement (unit angle), and a staying time rate of the gaze movement in each area which was de-fined as dividing the staying time in each area by the total staying time of all areas was used.

\subsubsection{Experimental B: Brain activity measurement and analysis during task execution}

NIRS captures the changes in hemoglobin concentration accompanying brain blood flow changes that are dependent on neural activity in the brain. Oxygenated hemoglobin (oxy-Hb) is considered the best indicator of blood flow changes [7]. In this study, therefore, oxy-Hb was taken to be the indicator of brain activity during tasks. The measurement sites were from the inferior frontal gyrus to the frontal gyrus, which are thought to be active during search behavior [13]. NIRS data for the patient $\Pi$, were collected and analyzed when the same experimental task as in Experiment A above was performed. The measurement device used was NIRS. The sampling rate was $50 \mathrm{~Hz}$. Mean oxy-Hb values were 


\section{Preliminary Study on the Assessment of Patients with Impaired Visuospatial Ability in Three-Dimensional Space Using a New Head Mounted Display System with Virtual Reality Technology}

collected for the analysis. Mean oxy-Hb was calculated for a time equivalent to 10 seconds during the task. The calculated values are shown up to the fourth decimal place. Comparison of the mean oxy-Hb values under two conditions during the task execution was statistically analyzed using the Wilcoxon signed rank test on a total of 8 channels.

\section{RESULTS}

\subsection{Clinical common assessment of USN}

The Patient I information revealed no problems with the visual field to perform the task. In Table 1, the Brunnstrom stage [14] was V for both the upper and lower extremities. The total Functional Independence Measure (FIM) [15] was 84. The Mini-Mental State Examination (MMSE) [16] was 26. In the line cancellation test on the BIT, the score was 24 out of a total of 36 .

Table 1 Patient characteristics.

\begin{tabular}{lllllccc} 
Subject & Age & Diagnosis Lesion & Br-UE & Br-LE & FIM & MMSE & $\begin{array}{c}\text { Cancellation test } \\
\text { (BIT) }\end{array}$ \\
\hline Patient I & 83 & cerebral hemorrhage & V & V & 84 & 26 & 24 \\
Patient II & 67 & $\begin{array}{l}\text { cerebral hemorrhage } \\
\text { V }\end{array}$ & V & 116 & 30 & 36 \\
\hline
\end{tabular}

$\mathrm{Br}$; Brunnstrom stage, UE; upper extremity LE; lower extremity

MMSE; Functional Independence Measure

* All subjects were female. The lesion was right sided.

As shown in Figure 4, the lines that were not cancelled were from the central to somewhat right areas. With the CBS score, there were six items of mild neglect on the left side, such as bumping into people or objects on the left, and the total score was 6 (Table 2).

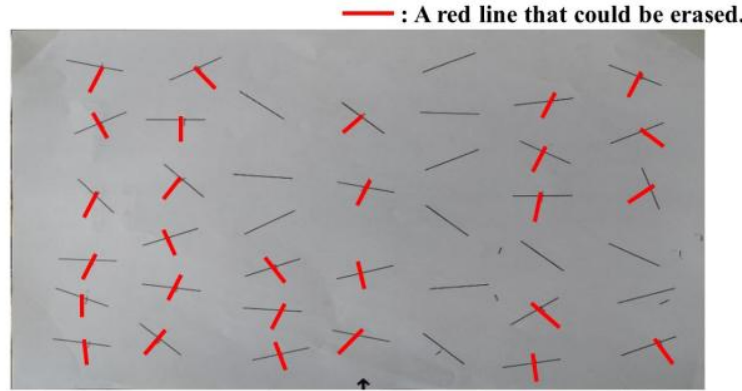

Fig. 4 The cancellation test of the patient $I$ as a clinical assessment (BIT) of USN.
Table 2. Catherine Bergego Scale of Patient I and Patient II.

\begin{tabular}{|c|c|c|}
\hline & Patient I & Patient II \\
\hline 1. Fails to groom or shave the left part of his/her face & $\square$ & $\square$ \\
\hline $\begin{array}{l}\text { 2. When dressing fails to adjust the left side correctly (sleeve, } \\
\text { slipper...) }\end{array}$ & 1 & 1 \\
\hline 3. Leaves food on the left side of his/her plate & & \\
\hline 4. Forgets to wipe the left side of his/her mouth after eating & . & 1 \\
\hline $\begin{array}{l}\text { 5. Exhibits difficulty in looking towards the left } \\
\text { 6. Ignores the left part of his/her body (e.g. does not put his/her }\end{array}$ & 1 & 1 \\
\hline $\begin{array}{l}\text { upper limb on the armrest, or his/her left foot on the wheelchair } \\
\text { footrest, or forgets to use his/her left arm when he/she needs to) }\end{array}$ & 1 & 1 \\
\hline 7. Does not notice or pay attention to noise or people addressing & & \\
\hline him/her from the left & 1 & 1 \\
\hline $\begin{array}{l}\text { 8. Collides with people or objects on the left side, such as doors or } \\
\text { furniture (when walking or in a wheelchair) }\end{array}$ & 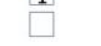 & $\square$ \\
\hline $\begin{array}{l}\text { 9. Has difficulty in finding his/her way towards the left when } \\
\text { moving }\end{array}$ & 1 & 1 \\
\hline about in familiar places or in the rehabilitation unit & 1 & 1 \\
\hline bathroom when they are on the left side & $(6 / 30)$ & $(7 / 30)$ \\
\hline
\end{tabular}

\subsection{Assessment of USN with HMD}

The object cancellation (Figure 5) rate was $100 \%$ for the patient I with both ego-centric coordinates. With object-centered coordinates, in places relatively close to the subject at $35 \mathrm{~cm}$, the rate was $60 \%$ in the left area and $100 \%$ in the central and right areas. At $45 \mathrm{~cm}$, it was $0 \%$ in the left area, about $80 \%$ in the central area, and $90 \%$ in the right area. At $55 \mathrm{~cm}$, it was $0 \%$ in the left area, about $20 \%$ in the central area, and $60 \%$ in the right area.

Figure 6 showed a staying time rate in each area for every $10^{\circ}$ of gaze movement, with the number of times the gaze stops to the left (negative direction) and right (positive direction), up to $55^{\circ}$. This was done with gaze movement, which is the total of eye movement and head movement, taken to be $0^{\circ}$ when gaze was directed at the center of the screen. The staying time rate in each area is defines as dividing the staying time in each area by the total staying time of all areas. The total range that can be seen on the HMD display is around $60^{\circ}$ to the left side from the right side. In the patient $\mathrm{I}$, the gaze movement was in a range of $55^{\circ}$ to the left and $35^{\circ}$ to the right with egocentric coordinates and objects were cancelled. The number of gaze stops was the highest at $15^{\circ}$ to the left and $25^{\circ}$ to the right. With object-centered coordinates, the gaze movement was $55^{\circ}$ to the left and $55^{\circ}$ to the right. The highest number of gaze stops was $55^{\circ}$ to the left.

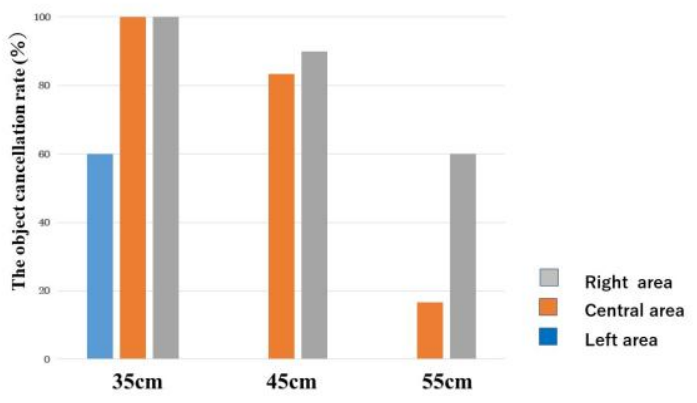

Fig. 5 The object cancellation rate of the condition of object-centered coordinates for the patient $I$ at 35,45 , and $55 \mathrm{~cm}$. 

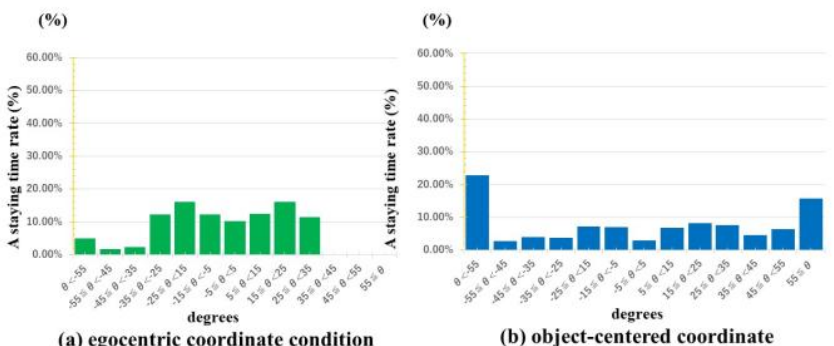

Fig. 6 A staying time rate for every 10degrees of the gaze movement, with the number of times the gaze stops to the left and right for the patient $I$ with egocentric and object-centered coordinates.

\subsection{NIRS measurement of brain activity during task} performance

The Patient $I$ information revealed no problems with the visual field to perform the task. In Table 1, the Brunnstrom stage was $\mathrm{V}$ for both the upper and lower extremities. The total FIM score was 116 . The MMSE was 30. In the line cancellation test on the BIT, the score was 36 . With the CBS score, there were three items of mild neglect on the left side, such as bumping into people or objects on the left, and the total score was 7 (Table 2). For the results of brain function measurement during the task trials, the mean oxy-Hb levels in the vicinity of the inferior frontal gyrus during task execution $(2 \mathrm{~min} /$ trial $\times 4$ trials) on the object cancellation test in this experiment were shown (Table 3). Table 3 gage the values in the vicinity of the inferior frontal gyrus on the left and right during task execution with the egocentric and object-centered coordinate systems in the patient $\Pi$. Except for the vicinity of the right inferior frontal gyrus, the values were higher with the object-centered coordinates. These values were significantly higher than the values at rest.

Table 3 The mean oxy-Hb during the task performance for the patient II with NIRS.

\begin{tabular}{cccccc}
\hline channel & \multicolumn{2}{l}{$\begin{array}{l}\text { egocentric } \\
\text { coordinate }\end{array}$} & \multicolumn{2}{l}{$\begin{array}{l}\text { objected-centered } \\
\text { coordinate }\end{array}$} & Pvalue \\
\hline $2 \mathrm{ch}$ & $0.1 \quad \pm 14.7$ & $0.2 \pm 4.2$ & $* *$ \\
\hline $8 \mathrm{ch}$ & $2.0 \pm 8.1$ & $3.5 \pm 19.1$ & $*$ \\
\hline
\end{tabular}

2ch; right inferior frontal gyrus $8 \mathrm{ch}$; left inferior frontal gyrus $\quad \mathrm{P}<0.5^{*}, 0.01^{* *}$ oxy-Hb; oxygenated hemoglobin $(\mathrm{mM} \cdot \mathrm{mm})$

\section{DISCUSSION}

According to the results of evaluation of USN with HMD, at three distances from the subjects, $35 \mathrm{~cm}, 45 \mathrm{~cm}$, and $55 \mathrm{~cm}$, the cancellation rate showed the same trend of left area < central area < right area. The cancellation rate was the lowest at the greatest of the three depth distances, $55 \mathrm{~cm}$. This can only be seen with three-dimensional VR space as in this experiment, as scoring cannot be done on the BIT line cancellation test since tests related to depth cannot be performed. Moreover, cancellation could not be done from the center to the right in BIT line cancellation, which was a divergence from the behavior of neglecting the left on CBS. This is a result that has not been obtained previously, in which neglect on the left as well as the furthest depth was exacerbated on this cancellation test and the CBS.

With gaze movement of the patient I, it was more movement that shifted the line of sight to the left spatial neglect region. In particular, a line-of-sight approach was adopted for attempting to look further to the left in the object-centered condition than in the egocentric condition. In addition, it was found that with object-centered coordinates, the object cancellation rate in subjects was lower with distal condition $(55 \mathrm{~cm})$ than with proximal condition $(35 \mathrm{~cm})$. It is said from other studies as well that the severity of neglect differs in proximal and distal space [17]. Our new method may be able to evaluate complex pathology of USN by analysis of versatile depth at two coordinates.

From NIRS measurements, it is generally known that the inferior frontal gyrus is activated during task-related searches [13], but in this study, greater brain activity was seen during the task than at rest in the patient with USN. Brain activity was also seen to be more active with object-centered coordinates than egocentric coordinates. This may have been because the HMD display allowed subjects to concentrate on the objects only, so that individuals' ability to concentrate was heightened by the simulation of a natural environmental condition, and the brain became more active. In addition, the patient's brain activity on the paralyzed side was low with object-centered coordinates and differences were seen with the healthy side. This patient's medical history included hemorrhage near the right frontal lobe, and so the result was low brain activity in the part near the paralyzed region. In the future, analysis of measurements over time of the activation status on the paralyzed side of the brain before and after patient rehabilitation may be a means to assess the effect of rehabilitation and estimate the patient's future recovery. It may also be necessary to investigate in detail the differences in activation of brain function with egocentric and object-centered coordinate conditions and multiple depth conditions, and clarify the pathological condition of patients' spatial neglect.

Compared with fMRI and other techniques, NIRS has the advantages of being smaller and more por and requiring less patient restraint, which make it possible to measure brain function during movement [18]. This is the reason it was used in the present experiment. With regard to the interpretation of signals obtained in NIRS, oxy-Hb is reported to be the most highly correlated with changes in local brain blood flow [19] and change in oxy- $\mathrm{Hb}$ is considered to be the parameter that reflects cerebral activation [18]. With NIRS, however, it is not possible to make detailed determinations of the localization of brain function, including in the deep brain, as can be done with fMRI, and so it is difficult to elucidate the pathological condition of spatial neglect with NIRS alone. In addition, what regular NIRS devices are able to measure is the amount of change in hemoglobin from baseline, and this needs to be combined with baseline measurements using other methodologies, such as PET. However, NIRS has a big advantage in that it is possible to measure brain activity during the execution of behavioral tasks in daily life more dynamically, which was difficult to do with previous brain function measurement methods such as PET or fMRI. In particular, its utility as an objective indicator of the functional recovery process in rehabilitation of patients with brain damage and others will probably increase in the future. This 


\section{Preliminary Study on the Assessment of Patients with Impaired Visuospatial Ability in Three-Dimensional Space Using a New Head Mounted Display System with Virtual Reality Technology}

study may contribute to a more detailed understanding of the pathological condition of neglect and its ap-plication to rehabilitation.

\section{CONCLUSION}

In this study, an assessment tool with the three quantitative measurement functions below was developed, and its validity was verified using patients with USN. (1) Quantitative assessment of cognitive regions in three-dimensional space using VR, (2) Quantitative assessment based on egocentric and object-centered coordinate theory, (3) Quantitative measurement of eye and head movement during object search. The results suggest the possibility of analyzing versatile pathological conditions of USN with newly HMD system. Moreover, the results of NIRS suggested that it may also be necessary to investigate in detail the differences in activation of brain function with egocentric and object-centered coordinate conditions and multiple depth conditions and clarify the pathological condition of patients' spatial neglect.

\section{ACKNOWLEDGMENT}

The authors would like to thank all subjects who kindly participated in the study.

\section{FUNDING}

This work was supported by JSPS KAKENHI Grant Number JP 17H02132, $20 \mathrm{H} 04049$.

\section{REFERENCES}

[1]. Japanese Ministry of Health, Labor and Welfare. 2017 https://www.mhlw.go.jp/stf/wp/hakusyo/kousei/18/backdata/01-01-0204.html

[2]. A Parton, P Malhotra, M Husain. Hemispatial neglect. Journal of Neurology, Neurosurgery \& Psychiatry 75(1):13-21, 2004.

[3]. Azouvi P, Samuel C, Louis-Dreyfus A, et al.: Sensitivity of clinical and behavioral tests of spatial neglect after

right hemi sphere stroke. J Neurol Neurosurg Psychiatry. 73: 160-166, 2002

[4]. T Ogourtsova, P Archambault, S Sangani, and A Lamontagne. Ecological Virtual Reality Evaluation of Neglect Symptoms (EVENS): Effects of Virtual Scene Complexity in the Assessment of Poststroke Unilateral Spatial Neglect. Neurorehabil Neural Repair. 2018 Jan;32(1):46-61

[5]. W. R Brain. Visual orientation with special reference to lesions of the right cerebral hemisphere. Brain, 64:244-272, 1941.

[6]. P Nachev1 and M Husain. Disorders of visual attention and the posterior parietal cortex. Cortex, 42: 766-773, 2006.

[7]. Y Hoshi, N Kobayashi, M Tamura. Interpretation of near-infrared spectroscopy signals: a study with a newly developed perfused rat brain model. J Appl Physiol, 190: 1657-1662, 2001.

[8]. M J Farah, J L Brunn, A B Wong, M A Wallace, and P A Carpenter. Frames of reference for allocating attention to space: Ev-idence from the neglect syndrome. Neuropsychologia, 28(4): 335-347, 1990.

[9]. Wilson B, Cockburn J, Halligan P. Development of a behavioural test of visuospatial neglect. Archives of Physical Medicine and Rehabilitation, 68: 98-102, 1987.

[10]. Azouvi P, Sylvie Olivier, Godeleine de Montety, Christiane Samuel, Anne Louis-Dreyfus, Luigi Tesio. Behavioral Assessment of Unilateral Neglect: Study of the Psychometric Properties of the Catherine Bergego Scale. Arch Phys Med Rehabil. 84:51-7, 2003.

[11]. T Ogourtsova, P Archambault, S Sangani, and A Lamontagne. Ecological virtual reality evaluation of neglect symptoms (EVENS ) : Effects of virtual scene complexity in the assessment of poststroke unilateral spatial neglect. Neurorehabilitation and Neural Repair, 2018, 32(1): 46-61.
[12]. T Tanaka, T Ifukube, S Sugihara and T Izumi. A case study of new assessment and training of unilateral spatial neglect in stroke patients: effect of visual image transformation and visual stimulation by using a head mounted display system (HMD). JNER. 2010, 7:20, 1-8.

[13]. Brunnstrom S. Motor testing procedures in hemiplegia : based on sequential recovery stages. Phys Ther. $1966 ; 46: 357-375$.

[14]. C V Granger 1, B B Hamilton, J M Linacre, A W Heinemann, B D Wright. Performance profiles of the functional independence measure. Am J Phys Med Rehabil. 1993 Apr;72(2):84-9.

[15]. Folstein MF, Folstein SE, McHugh PR. " Mini-Mental State” · A practical method for grading the cognitive state of patients for the clinician · J Psychiatr Res 12 ; 189-198 ，1975.

[16]. L Aimola, I Schindler, A M Simone, A Vennerid. Near and far space neglect: task sensitivity and anatomical substrates. Neuropsychologia 2012, 50(6): 1115-1123

[17]. Mesulam, M.M. Spatial attention and neglect: parietal, frontal and cingulate contributions to the mental representation and attentional targeting of salient extrapersonal events. Philos Trans R Soc Lond B Biol Sci. 354:1325-46, 1999.

[18]. Watanabe, E., Yamashita, Y., Maki, A., Ito, Y. and Koizumi, H., "Non invasive functional mapping with multi channel near infrared spectroscopic topography in humans", Neuroscience letters, Vol. 205, No. 1 (1996), pp. 41-44

[19]. Hoshi, Y, Kobayashi N. and Tamura, M. "Interpretation of near-infrared spectroscopy signals: a study with a newly devel-oped perfused rat brain model", J. Applied Physiology, Vol. 90, No. 5 (2001), pp. 1657-1662. 